\title{
Long-term follow-up after first-line bronchoscopic therapy in patients with bronchial carcinoids
}

\author{
Hes A P Brokx, ${ }^{1}$ Marinus A Paul, ${ }^{1}$ Pieter E Postmus, ${ }^{2}$ Thomas G Sutedja ${ }^{3}$
}

\begin{abstract}
${ }^{1}$ Department of Surgery, VU University Medical Center Amsterdam, Amsterdam, The Netherlands

${ }^{2}$ Clatterbridge Cancer Centre, Liverpool Heart \& Chest Hospital, University of Liverpool, Liverpool, UK ${ }^{3}$ Department of Pulmonology, Erasmus Medical Center Rotterdam, Rotterdam, The Netherlands
\end{abstract}

\section{Correspondence to} H A P Brokx, Department of Surgery, VU University Medical Center, P.O. Box 7057, Amsterdam 1007 MB, The Netherlands; Hes.Brokx@vumc.nl

Received 29 December 2014 Revised 8 February 2015 Accepted 23 February 2015 Published Online First 16 March 2015

\section{CrossMark}

To cite: Brokx HAP, Paul MA, Postmus PE, et al. Thorax 2015;70:468-472.
ABSTRACT

Background Carcinoid of the lung is considered to be a low-grade malignancy. A subgroup presents as an endobronchial tumour. Surgical resection is considered the standard approach because of its metastatic potential and the possibility of an iceberg phenomenon for the endobronchial subgroup. Advances in noninvasive and minimally invasive technologies seem to justify a more lung parenchyma-sparing approach. Methods In patients presenting with bronchial carcinoids, initial bronchoscopic treatment (IBT) is first attempted for complete tumour eradication and sufficient tissue sampling for the proper differentiation of typical (TC) versus atypical (AC) histological type. Furthermore in cases with postobstruction problems the desobstruction is aimed at improving the patient's condition and by that alleviate surgery if that is needed. High resolution CT is performed 6 weeks post IBT to determine local tumour growth. Surgical resection follows in case of extraluminal disease, residual carcinoid inaccessible for IBT, or late recurrences not salvaged by repeat IBT.

Results Minimum follow-up was 5 years from start of treatment for 112 patients (65 women, 47 men), with a median age of 47 years (range 16-77 years). Eightythree patients (74\%) had TC, and 29 (26\%) AC. IBT only was ultimately curative in $42 \%$ of the cases (47/112): 42 TC, 5 AC. Disease-specific mortality including surgical mortality has been $2.6 \%(3 / 112)$ in patients with extraluminal carcinoids (3 $A C$ ).

Conclusions IBT, if with unsuccessful rescue surgery, is justifiable with excellent long-term outcome. IBT made surgery unnecessary in $42 \%$ of the cases. Iceberg phenomenon and metastatic potential in this group of patients with bronchial carcinoids are clinically insignificant.

\section{INTRODUCTION}

Carcinoids represent only a small portion of all pulmonary tumours, that is, $2-5 \%$. They belong to the spectrum of neuroendocrine tumours consisting of tumourlets, typical carcinoids (TCs), atypical carcinoids (ACs), large cell neuroendocrine carcinomas and small cell lung cancers in which TCs represent the relatively benign and small cell lung carcinomas the most malignant entities of this spectrum. ${ }^{12}$ Within this group of carcinoids the initial presentation can be in the parenchyma or mainly in the airway, described as bronchial carcinoids.

TC shows relatively low malignant behaviour with excellent 5-year and 10-year survival rates of $87-100 \%$ and $82-87 \%$, respectively. In case of positive lymph nodes in TC, 5 -year survival rates

\section{Key messages}

What is the key question?

- Is initial bronchoscopic treatment strategy in patients with bronchial carcinoids justifiable?

What is the bottom line?

- Initial bronchoscopic treatment strategy made surgery unnecessary in $42 \%$ of the cases with excellent long-term outcome.

\section{Why read on?}

- Initial bronchoscopic treatment strategy needs to be implemented in the treatment of patients presenting with bronchial carcinoids.

are still up to $92-100 \%$. ACs have a higher malignant potential with significantly lower 5 -year and 10 -year survival rates of $56-75 \%$ and $35-56 \%$, respectively. In case of positive lymph nodes 5 -year and 10-year survival rates for ACs dropped to $25-$ $78 \%$ and $25-59 \%$, respectively. ${ }^{3-6}$

Distinction between TC and AC is based on the classification criteria by Travis et $a l,{ }^{7}$ which are based on a retrospective analysis of surgically resected specimens. Historically, surgical resection is considered the treatment of choice for both entities mentioned above. Lung parenchyma preserving surgical techniques, such as surgical bronchotomy and sleeve resections are clinically acceptable. ${ }^{458}$ Current advancements in diagnostic and therapeutic modalities and further understanding of tumour biology enable and encourage many to explore less or minimally invasive diagnostic and therapeutic techniques for optimal preservation of healthy lung tissue. ${ }^{7} 9{ }^{10}$ We have previously reported that initial bronchoscopic treatment (IBT) seems to be a justifiable approach for bronchial carcinoids instead of immediate surgical exploration and resection. ${ }^{1-13}$ Moreover, recent literature showed favourable results for bronchoscopic treatment (BT) as well, in carefully selected patients. ${ }^{14-16}$ In this study we report the long-term outcome of our IBT strategy in patients with centrally located bronchial carcinoids.

\section{PATIENTS AND METHODS}

We have outlined this approach in great detail in the aforementioned report. ${ }^{13}$ Figure 1 is a summary of our original IBT approach.

Our current approach is shown in figure 2 and the current approach, in brief, is as follows; 


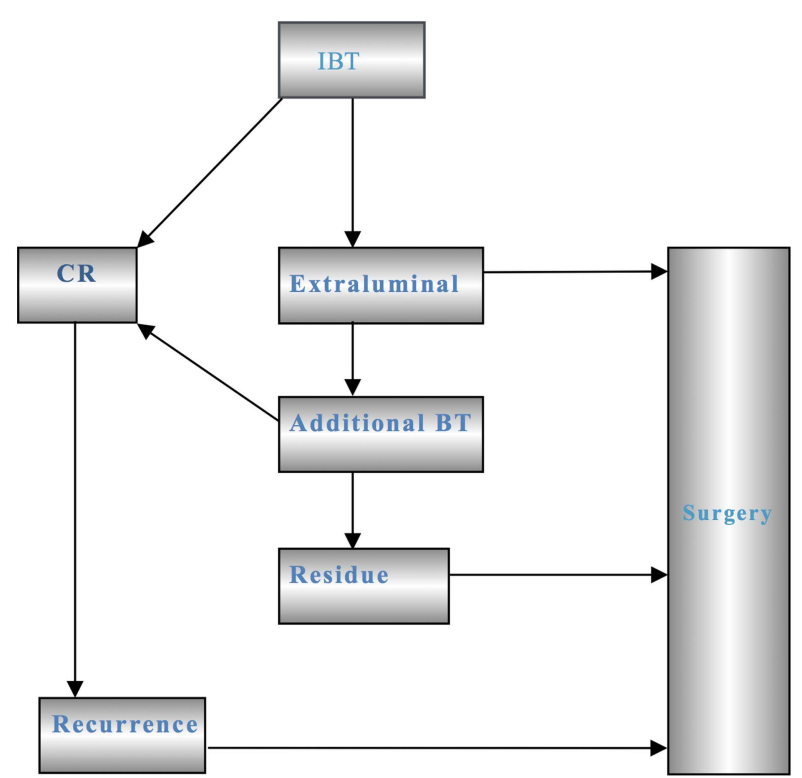

Figure 1 Original initial bronchoscopic treatment (IBT) strategy. CR, complete remission; BT, bronchoscopic treatment.

All patients referred to our institution for suspicion of harbouring a bronchial carcinoid undergo rigid and flexible bronchoscopy in one session for diagnostic purposes and complete resection of the intraluminal tumour growth. Six weeks post IBT for allowing postobstructive changes to resolve, a high resolution CT (HRCT) scan with intravenous contrast and repeat fibre-optic bronchoscopy is performed to assess IBT result. In case of residual extraluminal disease or AC histology or late recurrence not amenable for IBT, surgical resection is performed. In the later phase of this study period (since May 2000), AC successfully eradicated with IBT was subjected to close surveillance and not immediately followed by surgical resection. ${ }^{13}$ Successful IBT is defined if there is no sign of residual disease based on detailed bronchial wall images on thin slice HRCT with intravenous contrast and videobronchoscopy with negative biopsies. Close surveillance is by repeat HRCT and fibre-optic bronchoscopy 6-monthly for the first 2 years and annually up to 5 years; thereafter patients have been referred back to their pulmonologists with advice for yearly check-up. For intraluminal carcinoid recurrence during follow-up, BT is again attempted first to obtain tumour eradication prior to the decision for surgical resection.

We reviewed the prospectively gathered data of every referred patient to our centre-since the beginning of our protocolfrom 1991 till July 2009. This final analysis includes only those patients with at least 5 years follow-up after their first IBT. Every patient has been given detailed explanations of the pros and cons of our strategy. SPSS V.20 was used for data collection purposes.

\section{RESULTS}

From 1991 until July 2009, 112 patients have been accrued. For patients alive in July 2014 the median follow-up has been 112 months (range: 60-277 months). Four patients died within the first 5 years after inclusion. Patients' characteristics are shown in table 1, and IBT results in figure 3 and table 2.

A description of what is shown in figure 3 is further elucidated as follows.

In $37 / 112(33 \%)$ patients (33 TC, $4 \mathrm{AC}$ ) there was no sign of residual tumour (complete remission (CR)) after the first IBT session. In 45 patients (40\%: 29 TC, $16 \mathrm{AC}$ ) extraluminal growth was immediately apparent and surgical resection was performed. Of the remaining 30 patients (21 TC, 9 AC) with residual disease, 14 (11 TC, $3 \mathrm{AC}$ ) had residual intraluminal disease completely eradicated by repeating IBTs (range one to four sessions), one intraluminal residual carcinoid (TC) was inaccessible for IBT: this patient was unfit for surgical resection. The remaining 15 patients (9TC, $6 \mathrm{AC}$ ) had extraluminal disease ineligible for BT. Thirteen of them $(13 / 15 ; 87 \%)$ underwent surgical resection (7 TC, $6 \mathrm{AC}$ ). Three patients (3 TC) did not undergo surgery: one aforementioned patient had intraluminal residual disease inaccessible for IBT and unfit for surgery, and two patients had extraluminal residual disease and had refused surgery. All three are still alive and asymptomatic after IBT (follow-up being 140 months, 168 months and 205 months, respectively).

Fifty-one patients with CR after IBT (44 TC, 7 AC) have been closely monitored (table 3 ).

Six (4 TC, 2 AC) had local recurrences (12\%). In the two intraluminal recurrences (both TC) after 10 months and 63 months, repeat BT again resulted in CR. The remaining four
Figure 2 Current initial bronchoscopic treatment (IBT) strategy. $\mathrm{BT}$, bronchoscopic treatment.

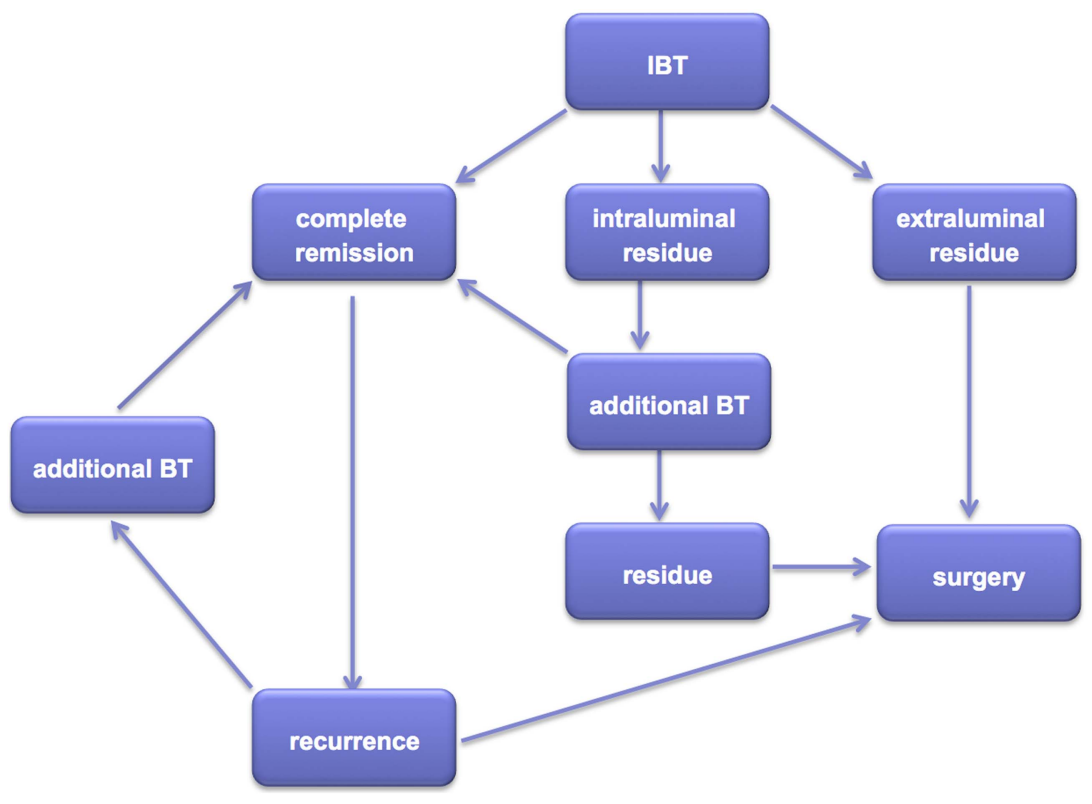


Table 1 Patient demographics at referral: bronchial carcinoid of the central airways

\begin{tabular}{lll}
\hline & Typical carcinoid & Atypical carcinoid \\
\hline Number of patients & 83 & 29 \\
Gender & & \\
$\quad$ Male & 37 & 10 \\
Female & 46 & 19 \\
$\quad$ Median age (range) in years & $47(17-77)$ & $48(16-67)$ \\
Smoking status & & \\
$\quad$ Non & 36 & 20 \\
Former & 30 & 7 \\
Current & 12 & 0 \\
Unknown & 5 & 2 \\
Presenting symptoms & & \\
Pneumonia (recurrent) & 31 & 13 \\
Haemoptysis & 16 & 6 \\
Asthmatic symptoms & 4 & 1 \\
Dyspnoea & 14 & 5 \\
Persisting cough & 12 & 4 \\
Other & 6 & 0 \\
\hline
\end{tabular}

(2 TC, 2 AC) developed extraluminal recurrences at 47 months, 104 months, 116 months and 198 months and underwent rescue surgery. It is important to point out that the extent of surgical resection would not have differed if patients had been operated at their first presentation prior to IBT despite the long interval period up to 198 months. For the final analysis, these four patients were grouped in the surgically resected cohort.

Sixty-two patients underwent surgical resection. We performed 5 pneumonectomies, 15 bilobectomies, one sleeve bilobectomy, 40 lobectomies (of which 15 were sleeve lobectomies) and one segmentectomy. For patients undergoing resections pTNM stages according to the 7th edition from the International Association for the Study of Lung Cancer of the TNM classification of malignant tumors were as follows: pT1aN0 (30 cases), pT1aN2 (2 cases), pT1bN0 (9 cases), pT2aN0 (7 cases), pT2bN0 (1 case); one case showed no residual disease. From 12 cases (all N0) the exact T-status could not be retrieved.

One patient who required emergency pneumonectomy for uncontrollable bleeding during IBT, recovered fully with specimen showing extraluminal TC N0. Remarkably, 139 months thereafter she presented with a solitary chest wall metastasis of a TC which was subsequently radically resected, and a dermal metastasis of the scalp developed (187 months postpneumonectomy) and was radically resected. She is now again free of disease for 8 months.

Metastatic disease developed in four patients (1 TC, $3 \mathrm{AC}$ ), two of them died. All had been treated with rescue surgery. One patient developed tracheal metastases of AC, repeatedly treated with IBT, and is alive. Overall distant metastases were seen in $1.2 \%(1 / 83)$ of TC and 10\% (3/29) of AC.

Twelve patients have died: eight TC, four AC (table 4). Two patients died because of metastatic extraluminal AC despite surgical treatment and one patient (AC) because of postoperative complications: that is, hypoxic encephalopathy after cardiac tamponade post lobectomy. The remaining nine patients died of unrelated causes; eight had only IBT and one rescue surgery. Disease-specific mortality including surgical mortality has thus been $3 / 112(2.6 \%)$. Overall disease-specific survival is $97 \%$. Within 5 years after IBT, four patients died of disease-unrelated and treatment-unrelated causes, a 5-year survival rate of $97 \%$ $(108 / 112)$. Disease-specific 5-year survival is $100 \%$ with our combined resection strategy. There were 60 patients with a follow-up of $>10$ years: 12 patients have died within this period; 2 because of the disease, 10 because of unrelated causes while 3 patients refusing surgery are still alive. Overall 10-year
Figure 3 Results of current initial bronchoscopic treatment (IBT) strategy in 112 patients with bronchial carcinoids. AC, atypical carcinoid; BT, bronchoscopic treatment; TC, typical carcinoid.

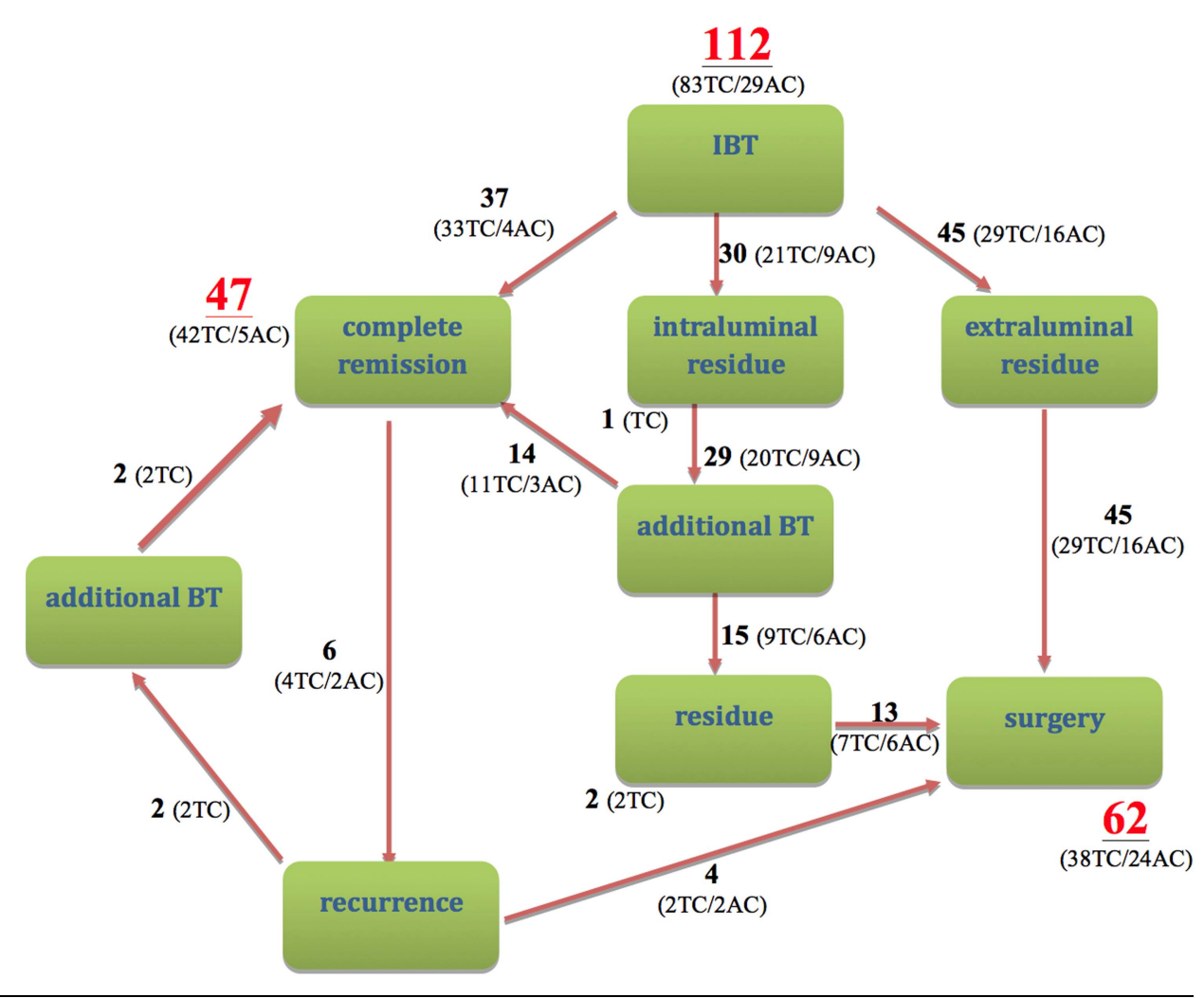


Table 2 Clinical outcome of patients with bronchial carcinoid after initial bronchoscopic treatment (IBT) with or without completion surgery

\begin{tabular}{|c|c|c|c|c|c|c|}
\hline Histology & $\mathrm{N}$ & Treatment & Outcome & Alive & Dead & Remarks \\
\hline \multirow[t]{4}{*}{ Typical (83) } & 43 & IBT & CR & 36 & 7 & All deaths unrelated \\
\hline & 3 & IBT & Residual & 3 & 0 & 1 unfit for surgery \\
\hline & & & & & & 2 refused surgery \\
\hline & 37 & Surgery & $\mathrm{CR}$ & 36 & 1 & 1 unrelated death \\
\hline \multirow[t]{4}{*}{ Atypical (29) } & 5 & IBT & CR & 5 & 0 & \\
\hline & 21 & Surgery & CR & 19 & 2 & 1 unrelated death \\
\hline & & & & & & 1 treatment-related death \\
\hline & 3 & Surgery & Metastatic & 1 & 2 & Hepatic and pulmonary metastases \\
\hline
\end{tabular}

No residual tumour detected macroscopically (videobronchoscopy, high resolution CT) and microscopically (biopsy and brush specimens).

$\mathrm{CR}$, complete remission.

survival is therefore $48 / 60(80 \%)$; disease-specific 10 -year survival rate is still $97 \%(58 / 60)$.

We compared tumour classification based on bronchoscopic versus surgical specimens in every surgically treated patient. In eight patients with extraluminal disease a shift from typical towards atypical histology classification was documented without any influence on treatment management and outcome.

\section{DISCUSSION}

The standard treatment for TC and AC is (surgical) resection. ${ }^{2-5} 17$ Carcinoid tumours harbour a malignant potential (AC being more malignant than TCs) and a 'tip of the iceberg' growth has been postulated in which the visible intraluminal part might just be a tiny fraction of tumour growing from deeper inside the tracheobronchial wall.

So, subjecting patients to non-surgical resection by less-invasive options, for example BT, was deemed unacceptable as this is not an approach through which resection margins could be checked for residual tumour; nor is sampling of lymph nodes possible. Surgical parenchyma-sparing techniques such as bronchotomy are, nevertheless, acknowledged to be acceptable from the oncological viewpoint; this is in contrast to surgery for non-small cell lung cancer. ${ }^{4} 8$

After achieving a long-lasting CR in a small group of patients with endobronchial TC after endobronchial resection ${ }^{11}$ we started a prospective study to diligently address the abovementioned issues. $^{11-13}$

Current long-term data clearly show the relatively low and slow malignant potential of centrally located bronchial carcinoids with only two deaths and one patient still alive despite extraluminal atypical metastatic disease after radical surgery.
One could suggest that delaying surgery by the described approach might induce rapid regrowth and lead to unresectability, more extensive surgery or fast distant spread; this however, is not supported by the observations in this study. There were six patients with local recurrences after IBT and in the four patients in whom surgical delay might have been caused, the extent of surgical resection and long-term outcome have not been compromised. Four patients with extraluminal disease on diagnostic HRCT performed 6 weeks after IBT and radically resected with N0 status still developed metastases later on. For the three patients not achieving CR after IBT, but not operated upon, survival with disease has been long.

Iceberg phenomenon can be clinically observed by looking at the detailed images of current HRCT in defining tumour growth pattern, in addition to bronchoscopic examinations in the vast majority of our cases. One would argue that too many repeat HRCTs and bronchoscopies are burdensome, causing long-term emotional distress and increased costs. A more detailed analysis about cost-effectiveness of IBT strategy is underway. Our unpublished data on the Hospital Anxiety and Distress Scale, showed no difference in scores for both group of patients. One may carefully state that the 'so-called' burden of repeat investigations and uncertainty does not seem to outweigh the $42 \%$ success rate of IBT as a complementary initial approach to surgical resection. At least to our knowledge, there has not been any publication to address this issue properly.

As a result of continuous reflection on optimising our IBT strategy diagnostically and therapeutically during this longitudinal study, back referral to the pulmonologists after successful IBT is now much earlier and follow-up has been scheduled less frequently, that is, HRCT repeat scan occurs at an interval of

Table 3 Follow-up (FU) of patients still alive in months from initial bronchoscopic treatment (IBT) until July 2014

\begin{tabular}{|c|c|c|c|c|c|}
\hline Carcinoid & Treatment (n) & Outcome (n) & Median FU (mo) & Range FU (mo) & Remarks \\
\hline \multirow[t]{7}{*}{ Typical (75) } & IBT (38) & CR (33) & 120 & $(73-241)$ & \\
\hline & & Residue (3) & 168 & $(140-205)$ & 1 unfit, 2 refused \\
\hline & & Recurrence (2) & 10 and 63 & & IBT-Recurrence \\
\hline & & & 98 and 29 & & BT_July 2014 \\
\hline & Surgery (37) & CR (35) & 129 & $(60-267)$ & \\
\hline & & Recurrence (2) & 47 and 104 & & IBT-Recurrence \\
\hline & & & 57 and 111 & & Surgery-July 2014 \\
\hline \multirow[t]{4}{*}{ Atypical (25) } & IBT (5) & CR (5) & 109 & $(83-170)$ & \\
\hline & Surgery (20) & CR (18) & 88.5 & $(60-159)$ & \\
\hline & & Recurrence (2) & 116 and 198 & & IBT-Recurrence \\
\hline & & & 113 and 79 & & Surgery-July 2014 \\
\hline
\end{tabular}


Table 4 Follow-up (FU), that is, survival (in months) of deceased patients with bronchial carcinoids

\begin{tabular}{lllrl}
\hline Histology & Treatment & Outcome & FU & Cause of death \\
\hline Typical (8) & IBT (8) & CR (8) & 2 & Pulmonary embolism \\
& & & 28 & Cardiovascular failure \\
& & & 68 & Unknown \\
& & & Respiratory insufficiency \\
& & & 93 & Sarcoma \\
& & & 140 & Natural cause \\
& & & 170 & Chronic myeloid leukemia \\
Atypical (4) & Surgery (4) & CR (2) & 24 & Hypoxic encephalopathy \\
& & & 66 & Murdered 'crime passionnel' \\
& & Metastatic (2) & 85 & Palliative sedation \\
& & & 114 & Pneumonia \\
\hline
\end{tabular}

No residual tumour detected macroscopically (videobronchoscopy, high resolution CT) and microscopically (biopsy and brush specimens).

$\mathrm{CR}$, complete remission; IBT, initial bronchoscopic treatment.

$>2$ years with virtual bronchoscopy assessment without repeat fibre-optic bronchoscopy in addition to the HRCT in case of no residual tumour or recurrence.

So far, in $73 \%(82 / 112)$ of the cases, the initial assessment has clearly indicated intraluminal versus extraluminal tumour growth. Furthermore, in the remaining 30 cases, the first 6 weeks' evaluation after IBT promptly indicated those who required surgical salvage immediately. The 6 weeks delay argument cannot justify immediate surgical exploration and resection given the perspective of successful long-term outcome after IBT in $42 \%$ of the cases (47/112). For the majority of the patients within 6 weeks period after accrual, the advice for treatment of choice is clear.

The long-term outcome also indicates that general concern for potential rapid regrowth after successful IBT in 57/112 $(51 \%)$ patients is largely exaggerated, albeit resulting in too frequent repeat HRCTs and bronchoscopic examinations during close surveillance in the study protocol. There were only $4 / 51$ $(7.8 \%)$ recurrences in the IBT group requiring surgery. They were diagnosed after 47-198 months follow-up, and surgical salvage was uncompromised.

Another central issue in the controversies, especially with the atypical variant is its malignant potential causing late recurrence beyond curative surgical option, in which many still advocate more aggressive treatment options. ${ }^{17}$ The Travis classification based on surgically resected specimens lacking data on tumour growth (ie, central vs peripheral/parenchymal) is possibly not optimal for the patients presenting with mainly endobronchial carcinoid. ${ }^{7}$ Nevertheless, current understanding and classification of bronchial carcinoids seem incomplete, and molecular characterisation of the resected specimens, especially in patients who showed a shift from typical to atypical for potential molecular drift is underway.

So far, our long-term results show that initial bronchoscopic assessment in exploiting the potential of intraluminal tumour eradication seems justifiable. The strategy offers clear and relatively rapid distinction towards the need for surgical salvage without compromising outcome. Several authors have additionally published their results of BT, although in smaller series but showing a similar trend. ${ }^{2}{ }^{14-16} 18$ It is important to stress bronchoscopic and surgical approaches are complementary to optimise management of these patients. Our IBT strategy proves to clearly determine whether there is need for surgery or that bronchoscopic therapy suffices. By doing so, a large percentage of patients presenting with bronchial carcinoids (42\%) can be treated curatively as well as safely in the most parenchymasparing way, without compromising outcome.

In conclusion, our long-term follow-up shows that IBT strategy in patients with bronchial carcinoids, complemented with surgical resection in case of extraluminal disease, resulted in excellent long-term outcome. Further studies of bronchial carcinoids' molecular heterogeneity seem warranted. IBT strategy showed to be a justifiable alternative for surgical resection in $42 \%$ of the cases, and needs to be implemented in the treatment of patients presenting with bronchial carcinoids.

Contributors All four authors contributed substantially to the conception and design of the work, acquisition, analysis and interpretation of data for this study. Every author revised this article critically for important intellectual content and gave final approval of the version to be published. Furthermore every author agrees to be accountable for all aspects of the work in ensuring that questions related to the accuracy or integrity of any part of the work are appropriately investigated and resolved.

\section{Competing interests None.}

Ethics approval Institutional review board.

Provenance and peer review Not commissioned; internally peer reviewed.

\section{REFERENCES}

1 Soga J, Yakuwa Y. Bronchopulmonary carcinoids: an analysis of 1,875 reported cases with special reference to a comparison between typical carcinoids and atypical varieties. Ann Thorac Cardiovasc Surg 1999:5:211-19.

2 Fink G, Krelbaum T, Yellin A, et al. Pulmonary carcinoid: presentation, diagnosis, and outcome in 142 cases in Israel and review of 640 cases from the literature. Chest 2001;119:1647-51.

3 Filosso PL, Rena O, Donati G, et al. Bronchial carcinoid tumors: surgical management and long-term outcome. J Thorac Cardiovasc Surg 2002;123:303-9.

4 Hage $R$, de la Riviere $A B$, Seldenrijk $C A$, et al. Update in pulmonary carcinoid tumors: a review article. Ann Surg Oncol 2003;10:697-704.

5 Detterbeck FC. Management of carcinoid tumors. Ann Thorac Surg 2010;89:998-1005.

6 Bertino EM, Confer PD, Colonna JE, et al. Pulmonary neuroendocrine/carcinoid tumors: a review article. Cancer 2009;115:4434-41.

7 Travis WD, Rush W, Flieder DB, et al. Survival analysis of 200 pulmonary neuroendocrine tumors with clarification of criteria for atypical carcinoid and its separation from typical carcinoid. Am J Surg Pathol 1998;22:934-44.

8 Stamatis G, Freitag L, Greschuchna D. Limited and radical resection for tracheal and bronchopulmonary carcinoid tumour. Report on 227 cases. Eur J Cardiothorac Surg 1990;4:527-32

9 Walch AK, Zitzelsberger HF, Aubele MM, et al. Typical and atypical carcinoid tumorsof the lung are characterized by $11 \mathrm{Q}$ deletions as detected by comparative genomic hybridization. Am J Pathol 1998;153:1089-98.

10 Skov BG, Krasnik M, Lantuejoul S, et al. Reclassification of neuroendocrine tumors improves the separation of carcinoids and the prediction of survival. J Thorac Oncol 2008;3:1410-15

11 Sutedja TG, Schreurs RG, Vanderschueren RG, et al. Bronchoscopic therapy in patients with intraluminal typical bronchial carcinoid. Chest 1995;107:556-8.

12 van Boxem TJ, Venmans BJ, van Mourik JC, et al. Bronchoscopic treatment of intraluminal typical carcinoid: a pilot study. J Thorac Cardiovasc Surg 1998;116:402-6.

13 Brokx HA, Risse EK, Paul MA, et al. Bronchoscopic treatment for patients with intraluminal bronchial carcinoids. J Thorac Cardiovasc Surg 2007;133:973-8.

14 Luckraz H, Amer K, Thomas L, et al. Long-term outcome of bronchoscopically resected endobronchial typical carcinoid tumors. J Thorac Cardiovasc Surg 2006:132:113-15.

15 Bertoletti L, Elleuch R, Kaczmarek D, et al. Bronchoscopic cryotherapy treatment of isolated endoluminal typical carcinoid tumor. Chest 2006;130:1405-11.

16 Fuks L, Fruchter O, Amital A, et al. Long-term follow-up of flexible bronchoscopic treatment for bronchial carcinoids with curative intent. Diagn Ther Endosc 2009;2009:782961.

17 Marty-Ane $\mathrm{CH}$, Costes V, Pujol JL, et al. Carcinoid tumors of the lung: do atypical features require aggressive management? AnnThorac Surg 1995; 59:78-83.

18 Katsenos S, Rojas-Solano J, Schuhmann M, et al. Bronchoscopic long-term palliation of a recurrent atypical carcinoid tumor. Respiration 2011;81:345-50. 